\title{
THE CONVOLUTION OF RADON MEASURES
}

\author{
H. KHARAGHANI
}

(Communicated by Richard R. Goldberg)

\begin{abstract}
The convolution of a pair of bounded Radon measures is extended to Cech-complete topological semigroups with separately continuous multiplication.
\end{abstract}

Let $S$ be a separately continuous topological semigroup, $C B(S)$ the space of all bounded real-valued continuous functions on $S$ and $M(S)$ the set of all bounded real-valued Radon measures on $S$. For $\mu$ in $M(S)$ and $f$ in $C B(S)$, a result of Glicksberg [3] implies that the map $t \rightarrow \int f(s t) d \mu(s)$ is continuous when restricted to the compacta of $S$. Hence it is $\nu$-measurable for any $\nu$ in $M(S)$. Let $\psi(f)=$ $\int\left[\int f(s t) d \mu(s)\right] d \nu(t)$ for $f$ in $C B(S)$. In this note it is shown that for a large class of semigroups, which includes the class of Cech-complete semigroups, $\psi$ is represented by a unique bounded Radon measure. Denoting this measure by $\mu * \nu$, we have

$$
\int f d \mu * \nu=\iint f(s t) d \mu(s) d \nu(t)=\iint f(s t) d \nu(t) d \mu(s)
$$

for each $f$ in $C B(S)$. This extends the convolution of a pair of bounded measures to a wide class of topological semigroups with separately continuous multiplication, including those considered in $[\mathbf{1}, \mathbf{3}, \mathbf{6}, \mathbf{7}]$.

All topological spaces are assumed to be completely regular Hausdorff. $\beta X$ denotes the Stone-Čech compactification of the topological space $X . f^{\prime}$ is the unique extension of $f$ in $C B(X)$ to $C(\beta X)$. For the set $A \subset X, \chi_{A}$ is the characteristic function of $A$. \|\| denotes the uniform norm.

LEMMA 1. Let $S$ be a completely regular separately continuous topological semigroup. Let $F, K$ be a pair of compacta of $S$, such that $K F$ is measurable in $\beta S$ with respect to every measure in $M(\beta S)$. Then for $\mu, \nu \in M(S)$ the functional, $\psi(f)=\int_{F} \int_{K} f(s t) d \mu(s) d \nu(t)$ for $f$ in $C B(S)$, is represented by a unique bounded Radon measure on $S$.

PROOF. It is sufficient to prove the lemma for $\mu \geq 0$ and $\nu \geq 0$. Let $\gamma^{\prime}$ be a regular finite Borel measure on $\beta S$ such that $\int_{\beta S} f^{\prime} d \gamma^{\prime}=\int_{F} \int_{K} f(s t) d \mu(s) d \nu(t)$ for $f$ in $C B(S)$. Let $C$ be a nonvoid compact subset of $\beta S-K F$. Let $A=\{f: f$ in $\left.C B(S), \chi_{C} \leq f^{\prime} \leq 1\right\}$. Index the elements of $A$ by $\left\{f_{\lambda}\right\}_{\lambda \in \Lambda}$ so that $\lambda>\eta$ iff $f_{\lambda}(x) \leq f_{\eta}(x)$ for each $x$ in $S$. Since the minimum of any pair of elements of $A$ is again in $A,\left\{f_{\lambda}\right\}_{\lambda \in \Lambda}$ is a (decreasing) net. For each $\lambda$ in $\Lambda$, let $g_{\lambda}(t)=$ $\int_{K} f_{\lambda}(s t) d \mu(s)$. Since $\mu \geq 0,\left\{g_{\lambda}\right\}_{\lambda \in \Lambda}$ is a decreasing net. By Urysohn's lemma

Received by the editors July 24, 1987.

1980 Mathematics Subject Classification (1985 Revision). Primary 43A05.

This work is supported by NSERC Grant A-7853. 
$f_{\lambda} \downarrow 0$ on $K F$ (note that the lemma is applied to the set $C,\{x\}$, where $x \in K F$, so that separate continuity suffices). Whence by Dini's theorem [5, p. 239] $f_{\lambda} \rightarrow 0$ uniformly on compacta of $K F$. Since $0 \leq f_{\lambda} \leq 1$ for each $\lambda, g_{\lambda}(t) \downarrow 0$ for each $t$ in $F$. A similar application of Dini's theorem implies that $\int_{F} g_{\lambda}(t) d \nu(t) \rightarrow 0$. Therefore $0 \leq \gamma^{\prime}(C) \leq \lim _{\lambda} \int_{F} \int_{K} f_{\lambda}(s t) d \mu(s) d \nu(t)=0$. Since $K F$ is $\gamma^{\prime}$-measurable in $\beta S$, $\gamma^{\prime}(\beta S-K F)=0$. Therefore $\gamma^{\prime}(\beta S)=\gamma^{\prime}(K F)$. Hence by [2, Corollary 4.4, p. 472] there is a unique bounded Radon measure $\gamma$ on $S$ such that $\int_{\beta S} f^{\prime} d \gamma^{\prime}=\int_{S} f d \gamma$ for each $f$ in $C B(S)$.

REMARK. The above proof remains intact if $K F$ is replaced by any subset $A$ of $S$ containing $K F$ which is measurable with respect to every measure in $M(\beta S)$. In this case the assumption that $K F$ is measurable is not needed.

THEOREM 2. Let $S$ be a completely regular separately continuous semigroup. Suppose for each pair of compacta $F, K$ of $S$ there is a subset $A$ of $S$ (depending on $F, K)$ such that $A$ is measurable with respect to every measure in $M(\beta S)$. Then the functional $\psi(f)=\iint f(s t) d \mu(s) d \nu(t)$ for $f$ in $C B(S)$ is represented by a unique bounded Radon measure on $S$ for any $\mu$ and $\nu$ in $M(S)$.

Proof. Let $\mu, \nu \geq 0$. For $\varepsilon>0$, let $F, K$ be a pair of compact subsets of $S$ such that $\mu(S-K)<\varepsilon, \nu(S-F)<\varepsilon$.

By Lemma 1 , there is a bounded Radon measure $\gamma$ on $S$ such that

$$
\int_{F} \int_{K} f(s t) d \mu(s) d \nu(t)=\int f d \gamma
$$

for each $f$ in $C B(S)$. Let $C$ be a compact subset of $S$ such that $\gamma(S-C)<\varepsilon$. Then $\left|\int f d \gamma-\int_{C} f d \gamma\right| \leq\|f\| \varepsilon$. Now

$$
\begin{aligned}
\left|\psi(f)-\int_{C} f d \gamma\right| \leq & \left|\iint f(s t) d \mu(s) d \nu(t)-\iint_{K} f(s t) d \mu(s) d \nu(t)\right| \\
& +\left|\iint_{K} f(s t) d \mu(s) d \nu(t)-\int_{F} \int_{K} f(s t) d \mu(s) d \nu(t)\right| \\
& +\left|\int_{F} \int_{K} f(s t) d \mu(s) d \nu(t)-\int_{C} f d \gamma\right| \\
\leq & \|f\| \mu(S-K) \nu(S)+\|f\| \mu(K) \nu(S-F)+\|f\| \varepsilon \\
< & \|f\|(\nu(S)+\mu(K)+1) \varepsilon .
\end{aligned}
$$

By [4, Proposition 4.1 and 2, Lemma 4.5] it follows that there is a unique bounded Radon measure on $S$, which will be denoted by $\mu * \nu$, such that $\psi(f)=\int f d \mu * \nu$ for each $f$ in $C B(S)$.

REMARKS. (a) For locally compact semigroups $S$ with separately continuous multiplication and for $\mu, \nu$ in $M(S)$, Glicksberg [3] proved that the map $t \rightarrow$ $\int f(s t) d \mu(s)$ is continuous on $S$ for each $f$ in $C B(S)$. This enabled him to obtain a bounded Radon measure $\mu * \nu$ such that $\int f d \mu * \nu=\iint f(s t) d \mu(s) d \mu(t)$ for each continuous $f$ vanishing at infinity. Later on Pym [6] and recently James C. S. Wong [7] proved that $\int f d \mu * \nu=\iint f(s t) d \mu(s) d \nu(t)$ for each $f$ in $C B(S)$. The assumption that $S$ is locally compact is essential in their proofs. Since a locally compact $S$ is open in $\beta S$, Theorem 2 implies all of the above. 
(b) H. Dzinotyiweyi [1] obtained a similar result for any topological semigroup with jointly continuous multiplication. The joint continuity is essential in his proof. Theorem 2 provides a new proof for his result.

A topological space $X$ is said to be Cech-complete if $X$ is a $G_{\delta}$ is $\beta X$. Examples of Cech-complete spaces include locally compact spaces and complete metric spaces. The following now is immediate from Theorem 2.

COROLlARY 3 . Let $S$ be a Čech-complete semigroup with separately continuous multiplication. Then for any $\mu, \nu$ in $M(S)$, there is a unique measure $\mu * \nu$ in $M(S)$ such that $\int f d \mu * \nu=\iint f(s t) d \mu(s) d \nu(t)$ for each $f$ in $C B(S)$.

Note that the Glicksberg result [3, Theorem 3.1] implies that $\iint f(s t) d \mu(s) d \nu(t)$ $=\iint f(s t) d \nu(t) d \mu(s)$ for each $f$ in $C B(S)$ for any topological semigroup $S$ and any $\mu, \nu$ in $M(S)$. (This follows from the middle inequalities in the proof of Theorem 2 and Glicksberg's result.)

ACKNOWLEDGEMENT. The author wishes to thank the referee for comments and suggestions.

\section{REFERENCES}

1. H. A. M. Dzinotyiweyi, Algebras of measures on $C$-distinguished topological semigroups, Math. Proc. Cambridge Philos. Soc. 84 (1978), 323-336.

2. R. A. Giles, A generalization of the strict topology, Trans. Amer. Math. Soc. 161 (1971), 467-474.

3. J. Glicksberg, Weak compactness and separate continuity, Pacific J. Math. 11 (1961), 205-214.

4. R. A. Hirschfeld, On measures in completely regular spaces, Bull. Soc. Math. Belg. 24 (1972), 275-287.

5. J. L. Kelley, General topology, Van Nostrand, Princeton, N.J., 1965.

6. John S. Pym, The convolution of functionals on spaces of bounded functions, Proc. London Math. Soc. (3) 15 (1965), 84-104.

7. James C. S. Wong, Convolution and separate continuity, Pacific J. Math. 75 (1978), 601-611.

Department of Mathematics, University of Alberta, Edmonton, Alberta, CANADA T6G 2G1 